far as it can be; the orbital margin is not broken; and though the bony portion of the lachrymal canal is cut this has not as yet given the patient any inconvenience. The only structure of importance that is seriously injured is the infraorbital nerve, which has all its branches severed either in the canal or immediately after their exit.

\section{PADDINGTON INFIRMARY.}

\section{A CASE OF SUBCUTANEOUS EMPHYSEMA IROM $\triangle$ RUPTURED} VOMICA.

(Under the care of Dr. SNELL.)

THAT this condition is a rare one is shown in the report of a case described by Mr. Shelswell and published in 'TFF LANCET of Dec. 21st, 1889. Dr. Snell confirms our observations as to the rarity of this complication and the paucity of the references to it which are to be met with in medical literature. Indeed, emphysema as an accompaniment of any form of disease of the air passages hardly receives attention in works on medicine, subcutaneous emphysema not being mentioned in the index of many of them. It has been described as following the excessive straining during parturition and also in whooping-cough in patients otherwise in good health, and has resulted from the perforation of an ulcer of the trachea or bronchi. Dr. Faggewrote: "A very rare effect of excavation of the lungs in the production of subcutaneous emphysema." Frantzel, alluding to it, wrote : "This subcutaneous empty. sema is sometimes observed in caseous pneumonia without pneumothorax in the case of a vomica reaching as far as the pleura pulmonalis, causing adhesions between this and the pleura costalis and perforating both pleural folds at once.' Dr. Brown has described two cases in which some of the air vesicles were ruptured in consequence of violent cough, and in which air escaped into the posterior mediastinum and thence spread all over the body. Both ended fatally.

A painter's labourer aged thirty-two was admitted on Jan. 9th of this year with symptoms of phthisis which had existed for eighteen months; there were physical signs of consolidation at both apices. The patient remained in the infirmary in very much the same condition until Feb 26 th. A more or less marked hectic temperature persisted during this period. On the evening of that day the right side of the face suddenly became swollen; this was found on examination to be due to subcutaneous emphysema; the next morning the neck was much swollen, the swelling extending over the whole of the chest anteriorly and posteriorly and in the lumbar region down to the iliac crests, also down the left arm to the wrist and down the right arm to the elbow. The percussion note at both apices was hyper-resonant; the breath-sounds could not be heard on account of the crepitations produced by the emphysema. On March 1st the emphysema was slightly, but not much, less. Between Feb. 26th and this date the hectic temperature had disappeared and the temperature had remained subnormal-it now rose to $101 \cdot 2^{\circ} \mathrm{F}$.; the pulse was 144 and of very low tension; the patient's condition was critical. The pupils were not dilated. On March 4th the emphysema was much less, but still present. The patient ditd on March 5 th.

The necropsy showed that there was no pneumo-thorax on either side; the upper lobe of the left lung was flled with tuberculous nodules of various sizes, the largest breaking down into purulent material; there was no perceptible cavity. The upper and middle lobes of the right lung were riddled with tuberculous nodules, there being some broken-down ones as large as filberts; a cavity of irregular shape as large as a walnut was found near the apex; the pleural surfaces at the right apex were excessively adherent The cavity above mentioned extended upwards by an irregular ulcerating track, which passed through the pleura at the apex and allowed a probe to be passed through it behind the first rib. The adhesions at the apex were very extensive and resistant No noticeable disease of the other organs existed.

Remarks by Dr. SNELL. - A somewhat similar case occurred at the Birmingham Children's Hospital and was reported in THE LANCET of April 29th of this year. Since the condition is barely mentioned in the text-books it is feasible to infer that the supervention of subcutaneous emphysema in phthisis must be a comparatively rare occurrence. When it does take place death appears to be imminent.

\section{BRECHIN INFIRMARY.}

AN EXTENSIVELY ADHERENT OTARLAN TLMOLR; REMOVAL ; RECOVERY.

(Under the care of Drs. MACKre and PARKrxsos.)

I' $T$ is extremely difficult to determine in any case of orarian tumour whether there are adbesions to surrounding parts or not. We must usually look to the history of the case for assistance in our diagnosis. If any statement is giren of attacks of abdominal pain, with or without romiting, then there is a strong probability that adhesions are present. This case is, however, an instructive example of the fact that we must not place too much reliance on the history; the fixity of the growth and the immobility of the abdominal wall were doubtless regarded as important signs in this patient. The pelvic adhesions are usually those which cause most difficulty to the operator and danger to the patient from fear of tearing the ureters, bladder, rectum or important vessels.

The patient, a multipara aged thirty-one years, her youngest child being eleven years old, was admitted into Brechin Infirmary on July 1st, 1893. A bout twelve months ago she felt a sharp pain in the hypogastric region and shortly afterwards observed a swelling in that region a little to the left of the median line. The swelling continued to increase rapidly and the pain, though not severe, was much aggravated by walking. For the past six months she had frequency of micturition; but apart from this there were no general or loca functional disturbances. Menstruation remained perfectly normal througbout the whole period. For the past month she had felt an uncomfortable stretching of the abdomen. Upon examination the abdomen was found to be much dis tended, bulging more to the right than to the left side. The subcutaneous abdominal veins were distended and the umbilicus was slightly retracted. The tumour, which con sisted of an elastic mass with distinct divisions, was quite immovable in any direction. In the act of taking a deep inspiration the abdominal wall did not move over the tumour The uterus was found to be firmly fixed, but its cavity was not encroached upon. There was no history of previous infam. matory attacks to indicate the formation of adhesions, on the 6 th chloroform was given and an incision of about four inclies was made over the tumour in the median line. As soon as the peritoneum was reached it was found that there were firm adhesions of that st ructure to the tumour midway between the umbilicus and symphysis and extending downward. Above this the omentum had an attachment for several inches. Three cysts were now tapped, their contents varying very much. Theadherent portion of the omentum was ligatured avd separated from the cyst with the knife. The adhesions to the anterior abdominal wall were torn through by manipulation. The tumour was now found to be firmly attached to the upper surface of the bladder, the upper and posterior surface of the uterus, to the whole of the right broad ligament and to the peritoneum in the region of the right sacro-iliac synchondrosis. To separate the tumour from the bladder was very difficult, and for a time it seemed that a portion of it would have to be ligatured and left attached; however, with care the adhesion was separated. The attachment to the uterus, being so extensive, could not fail to cause considerable injury to that organ in its separation. The right ovarian artery was ligatured and the right broad ligament removed with the tumour. The other peritoneal attachments were not difficult to overcome. The abdominal cavity was carefully washed out with weak carbolic lotion, a drainage-tube was put in and the abdominal incision was closed. For two days there was a little sickness, which, however, did not trouble the patient after that time. The day after the operation the temperature rose to $100.2^{\circ} \mathrm{F}$., but fell to normal in the evening; this morning rise and evening fall continued for five or six days. On the sixth day the temperature rose to $100.4^{\circ}$, but after this it gradually fell. Twenty-one days after the operation the patient was able to rise and at the present time she is perfectly well.

Remarks by Dr. MAckIE.-The chief interest of this case lies in the extensive adhesions and the good recovery after such extensive separation. It again illustrates how well patients bear abdominal operations when thorough antiseptic precautions are carried out. 